\title{
Opportunistic Strategies for Lightweight Signal Processing for Body Sensor Networks
}

\author{
Edmund Seto ${ }^{\dagger}$, Eladio Martin ${ }^{\ddagger}$, Allen Yang ${ }^{\ddagger}$, Posu Yan ${ }^{\ddagger}$, Raffaele Gravina* ${ }^{*}$ Irving Lin ${ }^{\ddagger}$, \\ Curtis Wang ${ }^{\ddagger}$, Michael Roy ${ }^{\ddagger}$, Victor Shia ${ }^{\ddagger}$, Ruzena Bajcsy ${ }^{\ddagger}$ \\ ${ }^{\dagger}$ School of Public Health, University of California, Berkeley; CA 94720, USA \\ ${ }^{\ddagger}$ Electrical Engineering and Computer Sciences, University of California, Berkeley; CA 94720, USA \\ * Wireless Sensor Networks Lab, Telecom Italia, Berkeley, CA 94720, USA and Department of Electronics, \\ Informatics, and Systems, University of Calabria, Rende, Italy
}

\begin{abstract}
We present a mobile platform for body sensor networking based on a smartphone for lightweight signal processing of sensor mote data. The platform allows for local processing of data at both the sensor mote and smartphone levels, reducing the overhead of data transmission to remote services. We discuss how the smartphone platform not only provides the ability for wearable signal processing, but it allows for opportunistic sensing strategies, in which many of the onboard sensors and capabilities of modern smartphones may be collected and fused with body sensor data to provide environmental and social context. We propose that this can help refine data reduction at the local level. We describe three examples related to health and wellness, to which our system has been applied.
\end{abstract}

\section{Categories and Subject Descriptors}

J.3 [Computer Applications]: Life and Medical Sciences-health, medical information systems.

\section{General Terms}

Design, Experimentation, Human Factors

\section{Keywords}

Wireless sensors, body area networks, opportunistic sensing, health monitoring, wearable computing, energy expenditure, physical activity

\section{INTRODUCTION}

Wireless body sensor networks (BSN) have numerous applications to health and wellness monitoring. The typical usage scenario involves an individual wearing a sensor layer - wireless sensors that monitor various aspects of vital signs, physiology, and/or motion at multiple locations on the body. Data from the sensor layer are transmitted to a network layer, which is responsible for transmitting the data (e.g., through WLAN, cellular, GPRS, or $3 \mathrm{G}$ wireless network) to a third, service layer.

Permission to make digital or hard copies of all or part of this work for personal or classroom use is granted without fee provided that copies are not made or distributed for profit or commercial advantage and that copies bear this notice and the full citation on the first page. To copy otherwise, or republish, to post on servers or to redistribute to lists, requires prior specific permission and/or a fee.

PETRA 2010, June 23-25, 2010, Samos, Greece.

Copyright 2010 ACM 1-58113-000-0/00/0004 ..\$5.00.
The remote service layer could be simply a database or more complex server application (e.g., an electronic medical records (EMR) system) at a healthcare provider's facility, which is tasked with processing the data into quality information that will be used to provide better patient care. A number of research projects follow this paradigm (e.g., MIThril, CodeBlue, ActiS) $[5,9$, 12]

Managing large volumes of data from BSN remains a challenge for practical applications of this technology - particularly those which require continual monitoring of individuals. It is inefficient to transmit raw sensor data to, and maintain data at the service level. Moreover, such an approach presents problems due to limited wireless bandwidth, the need to conserve power, considerable noise in raw sensor data, and the need for maintaining reliable and secure communication. This has motivated lightweight signal processing strategies, in which some processing of sensor data can be done locally to reduce many of the inherent problems associated with sending large amounts of raw sensor data to be remotely processed.

In this paper we describe our experience with integrating components into a heterogeneous BSN platform, and the hierarchical system of lightweight signal processing employed in our platform. At the lowest level of our system, raw sensor signals are processed by sensor motes before being sent to a mobile coordinator, which conducts further processing before sending information on to higher service-level applications. We present the latest developments of our BSN platform, which is now based on a smartphone running the Android operating system. We propose that the use of a smartphone as a BSN coordinator allows for an opportunistic sensing approach, in which many of the onboard sensors and capabilities of modern smartphones can provide useful environmental and social context to other BSN data, which allows for further data refinement.

\section{SYSTEM ARCHITECTURE}

At the outset, our research goal was to have BSN platform which was robust enough to be applied to numerous applications (e.g., patient monitoring, telemedicine, tele-rehabilitation, personal wellness, emergency response, studies of exposures to environmental hazards) and use by different populations (e.g., the elderly, children, patients with pre-existing conditions). This required a system architecture that could support a heterogeneous set of sensors that could be dynamically configured over-the-air for different application needs, and be appropriate for long-term monitoring applications in both indoor and outdoor environments [11]. 
Figure 1 illustrates the 3-layer hierarchy of our system, which we call DexterNet. At the body sensor layer (BSL) are sensor motes, which can consist of a combination commercially-available or custom sensor boards attached to motes (e.g., TelosB, Shimmer) worn on the body. These sensor motes communicate with the personal network layer (PNL), which consists primarily of a mobile base station (e.g., a PDA, Internet Tablet, or smartphone) that acts as a higher-level data and communications coordinator, and allows for user-interaction with the BSN. The base station coordinates communication with higher level application services that lie at the global network layer (GNL).

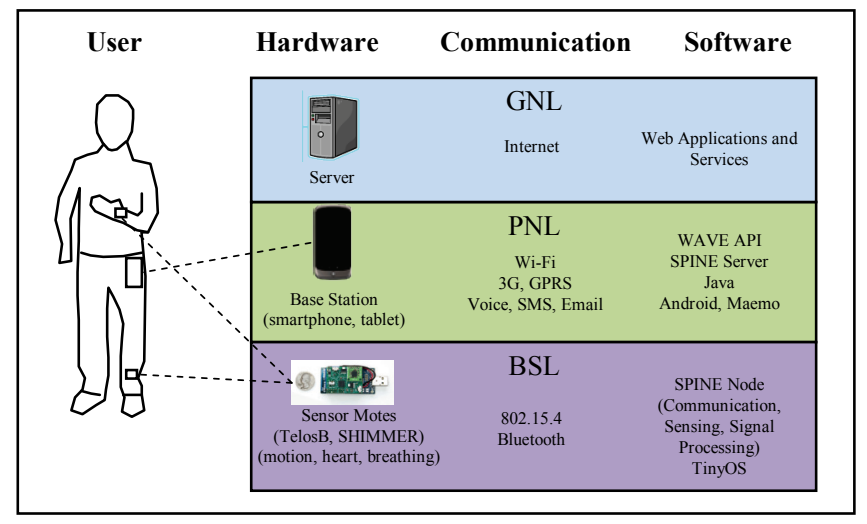

Figure 1. Three-layer architecture of the DexterNet system with example hardware, communication and software implementation.

\subsection{Hardware}

We have integrated various hardware devices to realize the BSN system. For motion classification research, we have integrated a custom-made sensor board with triaxial accelerometer and biaxial gyroscope on a TelosB mote. The mote has a 802.15.4 radio for wireless communication to a base station. For physical activity and health monitoring research, we have collaborated with researchers from the Tampere University of Technology to integrate a sensor board for heart ECG and electrical impedance pneumography for breathing measurements [14]. Most recently we have integrated the Intel SHIMMER mote and its associated motion and physiology sensors, via the Bluetooth radio.

At the personal network layer, we have collaborated with researchers from the University of Texas Dallas, to integrate the Nokia N800/N810 Internet Tablet as the base station for our system. The tablet has allowed the fusion of GPS measurements for outdoor localization of BSN data, as well as a commercial air pollution monitor (connected via serial cable) for research on personal exposures to air pollution and respiratory health. Additionally, we have begun to use smartphones running the Android operating system as our mobile base station, which has provided additional capabilities as described below.

\subsection{The SPINE framework}

DexterNet makes use of the open-source Signal Processing in Node Environment (SPINE) framework, which provides flexibility in integrating different sensors, processing algorithms, and communications for on-the-fly reconfiguration of the BSN [7]. SPINE consists of on-node TinyOS code that performs basic sensing and communication functions, as well as low-level signal processing of raw sensor data. Sensors and processing can be turned on or off over-the-air depending on different application needs. SPINE provides basic feature extraction algorithms for low-level processing of sensor data, including min, max, mean, variation, and energy. These features, as well as other algorithms which can be easily developed and added to SPINE, are computed on a sliding window of sampled sensor data, and are sent to the SPINE server module. Shifting some processing to the BSL nodes, may reduce on radio communication with PNL, thereby realizing benefits of lightweight signal processing.

In SPINE, a server module, implemented in Java acts as the coordinator for the BSN. We ported SPINE server to run on the Nokia N800 device, and are currently implementing a port to Android smartphones. As an application on the PNL mobile device, SPINE server can communicate with the sensor motes to reconfigure sensing and low-level processing. Higher level processing of either the raw sensor data or extracted features can be implemented in Java within a SPINE server application. Such an application may fuse data from various body sensors, as well as sensors onboard or attached to the mobile device. By making use of the higher processing and storage capability of the mobile device, higher level data filtering and classification algorithms are possible, potentially reducing the amount of communication with the GNL.

\subsection{Android and WAVE}

Android is an operating system for mobile devices. Android includes not only an operating system, but also a Java-based software development kit, and a set of mobile applications. One of the benefits of porting SPINE server and our BSN platform to Android is the large number of consumer mobile devices that are coming to market based on this operating system, which will aid in the translation of BSN research to practice. Additionally, because source code is available (e.g., for the Bluetooth radio library), Android is compatible with our overarching goal of creating a robust platform for heterogeneous BSN.

Current mobile devices running Android provide a number of rich sensing and interactive capabilities that can be integrated into BSN applications. For instance, current Android mobile phones (e.g., Google Nexus One) provide voice and sound, SMS, and data communication; camera, light, touch, vibration, text input, visual graphic display, GPS localization, and accelerometer.

In our port of the SPINE server to Android, we recognized the increasing ability for smartphones to provide sensor data and social interaction between users. To create a framework for integrating BSN data with these capabilities, we developed the WAVE application programming interface (API) for Android [18]. In essence, the WAVE API serves as Android glue between the BSL and GSN layers. WAVE provides application developers with a set of Java classes that simplifies the setup, access, and processing of BSN data, and integrates BSN with local and/or remote databases. The databases can be populated with data from multiple users, allowing for interactive BSN applications. WAVE is run as a service on the mobile device, and can be called by different Android applications that need to access the BSN or the databases.

From the standpoint of human factors, the Android mobile phone interface provides the user with a familiar device to interact with the BSN. Specifically, Android allows for integration of a BSNrelated application with other application and services on the 
phone through message calls, or intent objects. For example, the native web browser, SMS message, or telephone dialer applications can all be called from a BSN application. Moreover, because the mobile phone is a pervasive device, a user is more likely to carry it and maintain its battery because it provides many capabilities, as opposed to a dedicated device for health monitoring alone.

\section{APPLICATIONS}

We present three applications built on the DexterNet platform. The first is system for monitoring exposures to environmental pollutants. The second is a system for monitoring congestive heart failure patients. The third is a system for monitoring physical activity for obesity intervention.

\subsection{Environmental health monitoring}

The heterogeneous sensing model of DexterNet is well-suited to monitoring a user's exposures to environmental pollutants. Our system fuses motion, air quality, and location sensing, to build a rich database that has been used to characterize the magnitude of a person's exposure, as well as the underlying context of when, where, and why a person experiences his or her exposures [15]. Such a system has uses in the management of asthma and other respiratory health conditions.

In experiments of our system, individuals each wore five motion sensing motes placed on both wrists and ankles, and additionally at the front waist position. A Nokia N810 served as the base station, collecting GPS time and location measurements. A mote was also used to monitor heart and breathing variables. Additionally, a commercial airborne particulate monitor was connected via serial port to the Nokia. Individuals were asked to walk a prescribed route around the University of California, Berkeley campus, which included part of downtown, small and large arterial streets, as well as the center of campus, which was largely free of vehicle traffic. Figure 2 illustrates the results of one of the walks, which provides a multivariate perspective of the person's urban air pollution exposure experience. For this work, we developed and validated a lightweight energy expenditure algorithm in SPINE called Kcal.

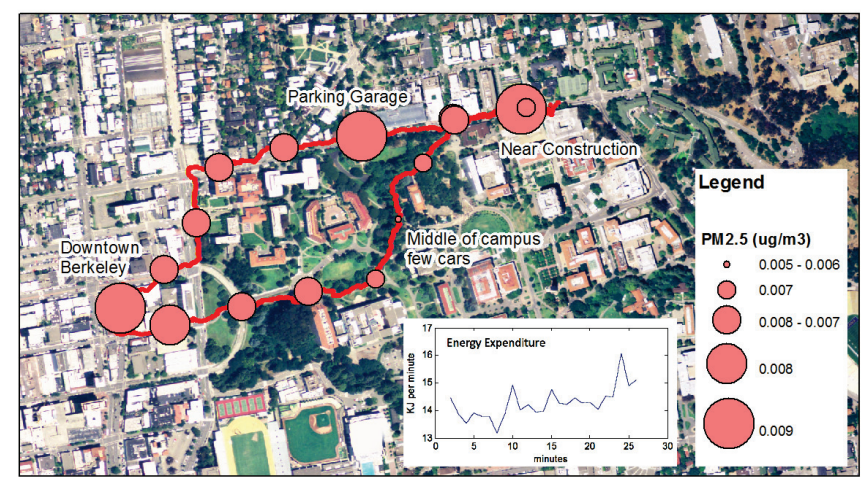

Figure 2.DexterNet system used to monitor exposure to urban air pollution.

\subsection{Congestive heart failure patient monitoring}

The heterogeneous sensing model of DexterNet can also be applied to telemedicine patient monitoring model, in which a patient is continually monitored, with data being set to an EMR system, which can then provide feedback to patients. In collaboration with Vanderbilt University researchers, we created a mobile system based on DexterNet for managing congestive heart failure.

The system consisted of a Nokia N810, connected via 802.15.4 radio to a motion mote located at the waist to monitor physical activity, and connected via Bluetooth to a digital scale and blood pressure monitor to monitor daily changes related to fluid overload and possibly changes due to blood pressure medication.

We tested the system in an experiment in which a user at Berkeley wore the system as part of their daily activity. In the morning, the user activated the system. When blood pressure and weight monitors were used, their measurements were automatically stored at the base station. During the day, the system continually computed and stored physical activity according to the Kcal energy expenditure algorithm in SPINE. When the mobile system found a Wi-Fi access point, the data were uploaded using standard Internet security protocols to a remote EMR at Vanderbilt. At the end of the day, the user received an SMS message as feedback, which suggested changes in behavior, such as increasing activity and/or changing doses of medication.

\subsection{CalFit for obesity intervention}

Physical activity is associated with numerous health outcomes. Most directly, it is part of the energy balance equation that is associated with the risk of obesity. Using DexterNet and the WAVE API for Android smartphones, we developed the CalFit application - a multi-user mobile application that monitors physical activity, and encourages exercise through social interaction and competition [18].

The current version of CalFit relies exclusively on the onboard sensing capabilities of smartphones like Google's Nexus One and Motorola's Droid. The triaxial accelerometer is used with the SPINE Kcal algorithm for energy expenditure. The algorithm was designed to be insensitive to sensor orientation. Hence, it can continually track activity even with the mobile phone arbitrarily placed in the user's pocket. On-board GPS is used to track the time and location of activities. The WAVE API allows storing workout data to remote servers, where it can be further managed and shared. Finally, Android intent objects are used to call the native SMS application so that workout data can be shared among users in a competitive fashion (Figure 3).

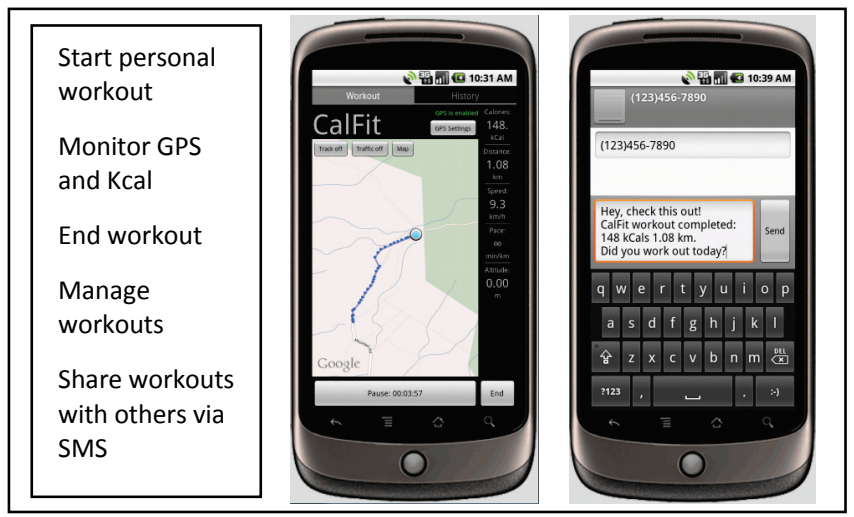

Figure 3. CalFit Android application flow. 
We are currently in the process of conducting user experiments with CalFit. Our hypothesis is that users will not only be motivated to increase physical activity by seeing their own progress, but competitive interaction with other users will also provide as motivation.

CalFit can serve as the foundation for further BSN research. We have begun testing the interface between SHIMMER motes and CalFit, with the goal of incorporating more sophisticated motion classification [19] into the system.

\section{OPPORTUNISTIC SENSING FOR BSN}

As mentioned above, consumer smartphones increasingly offer different sensing and communication capabilities. When integrated with BSN, we propose that there are numerous opportunistic sensing strategies that are consistent with the goals of lightweight sensing in terms of data reduction and refinement at the local PNL level.

Opportunistic sensing has been described as the tasking of mobile devices to collectively sense environmental data - the context in pervasive computing applications (e.g., in people-centric urban sensing) [3]. It shares similarities with cooperative sensing networks, in which distributed sensors are used in a cooperative fashion to provide greater fault tolerance, improved coverage and sensing quality, while leveraging of lightweight sensing devices [1]. However, the opportunistic sensing model typically describes a cooperation at higher levels (i.e., between people) than between multiple BSN devices worn on a single person.

\subsection{Location-based approaches: GPS and indoor localization}

Most mobile devices include localization in the form of GPS. Indeed in our work, we have extensively included GPS measurements to provide context for BSN data. Recent studies to monitor mobility and location with smartphones make use of GPS and accelerometer data [13]. Nevertheless, the location information obtained through GPS is only reliable in certain outdoor environments, and the accelerometer data are generally too noisy to obtain accurate information.

Reference [16] shows the importance of indoor localization, especially for hospitals and government offices. There have been several attempts to obtain indoor localization through image matching techniques $[10,6]$, but indoor environments are extremely challenging for this kind of application, and even if it is possible to recognize the shapes of pedestrians and objects through image processing techniques, the identification of similar ones is difficult for a computer vision-based system intended to monitor whole buildings [2]. In fact, the computational cost of high-dimensional feature extraction and processing is typically prohibitive for implementation in a cell-phone [8]. Consequently we have tried to focus on alternative ways for indoor localization.

Android is well-suited for localization due to its support of diverse sensors and hardware which enable localization (e.g. GPS, Wi-Fi, cellular communications, accelerometer), and its higher efficiency in comparison with other platforms such as J2ME [4]. In this sense, recent research work [17] presents location-based services running on Android based largely on outdoor GPS use, but precise indoor localization would be a valuable addition.
We have been exploring indoor localization based on the possibilities afforded by three smartphone resources: Wi-Fi radio, cellular communications radio and accelerometer, with the intention to build a multimode approach for localization. Making use of RSSI information from Wi-Fi beacons deployed within buildings, it is possible to obtain a radio map of different locations (fingerprinting), which will allow estimating locations through the comparison of the current RSSI measurements with those stored in the radio map. Processing this information statistically, we have obtained accuracies in the order of a meter and with real-time dynamicity (refreshment of location information every second). Nevertheless, we have found a major challenge with some smartphones (early generations of smartphones regardless of their Operating System), in the sense that the RSSI values were not refreshed dynamically. Smartphones running on Android 2.0 and above do not have this issue.

RSSI information from cellular base stations could theoretically be used to disambiguate locations for which the Wi-Fi radio map offers doubts. Nevertheless, we have found this approach unfeasible with current state of the art smartphones, because the refreshment rate of RSSI values is very slow (not dynamic enough for indoor walking) and the granularity in the RSSI values is poor and hardware dependent (e.g. G1 phone only distinguishes between 4 bars of coverage, and Droid only provides a few more intermediate values). Moreover, we could only read RSSI information from neighboring base stations belonging to the same SIM card operator, constraining the practicality of this approach.

While there are still challenges to realizing indoor localization on today's devices, the ability to use location in an opportunistic way to triage signal processing algorithms is promising. For example, in applications of continuous activity classification, we could first threshold on accelerometery to determine motion yes/no, and if yes, use localization to determine the context for the motion. If for instance, the user is moving and outdoors on a sidewalk, we could activate a step counting algorithm, or moving fast in the middle of a roadway we could instead activity a carbon footprint algorithm, or indoors in a stairwell, activate an algorithm to count stair climbing/descending. Triaging in this manner could greatly decrease the complexity of classification tasks, particularly given SPINE's capabilities for over-the-air reconfiguring of sensors and algorithms.

\subsection{Other approaches}

Even simple opportunistic approaches, such as using the clock on the smartphone may provide context to body sensor applications. In the case of our congestive heart failure project, a typical protocol may include only recording morning and evening weight and blood pressure, whereby the Bluetooth radio could be turned off most of the time to conserve power.

For environmental pollution monitoring where it is only necessary to sample while the user is outdoors, the mobile phone's light sensor may be used to discriminate indoor from outdoor conditions, and if outdoors, conduct environmental monitoring. Similarly, in lieu of a costly air pollution monitor, it may be possible to discern when a user is outdoors, and only then, sample sound from the phone's microphone. These sounds could then be used in vehicle sound detection algorithms and fused with BSN motion classification to quantify exercise and mobile source air pollution together. 
Additionally, the persistent connectivity of smartphones may lead to opportunistic strategies for BSN. For example, in CalFit, we will evaluate the degree to which an SMS message from one user to another triggers increased workouts in the other user. We may also evaluate whether Google Latitude, a location-based service for identifying nearby friends made possible by persistent Internet connectivity may lead to increased walking to meet with friends.

\section{CONCLUSIONS AND FUTURE WORK}

In this paper we have provided a description of a heterogeneous BSN system, which provides for a hierarchical structure of signal processing on-node and on a mobile base station. We have provided an overview of its application to three case studies. Finally, we have discussed the most recent evolution of our work to integrate an Android smartphone as the base station coordinator, which provides for opportunistic sensing strategies.

Moving forward, our group will continue the migration of our system to Android devices, and conduct user studies to evaluate the performance of the BSN. Our CalFit application will provide a foundation for future evaluation of BSN activity classification algorithms. In developing these algorithms, we will explore the tradeoffs between on-node versus base station processing. Additionally, we will evaluate the performance gains possible through our proposed BSN opportunistic sensing strategies.

\section{ACKNOWLEDGMENTS}

We thank Roozbeh Jafari for this encouragement and advice. We thank Ville-Pekka Seppä for his collaboration with our group to integrate his heart and respiration sensor. We thank Akos Ledecsi, Janos Sztipanovits, and Yuan Xue for their collaboration on the congestive heart failure project.

\section{REFERENCES}

[1] Agre J. and Clare, L. 2000. An Integrated Architecture for Coorperative Sensing Networks, Computer, 33, 5, 106-108.

[2] Ahn, H., and Yu, W. 2009 Environmental-adaptive RSSIbased indoor localization. IEEE Transactions on Automation Science and Engineering, October 2009, 6, 4, 626-633.

[3] Cornelius, C., Kapadia, A., Kotz, D., Peebles, D., Shin, M., and Triandopoulos, N. 2008. Anonysense: privacy-aware people-centric sensing. In Proceeding of the 6th international Conference on Mobile Systems, Applications, and Services (Breckenridge, CO, USA, June 17 - 20, 2008). MobiSys '08. ACM, New York, NY, 211-224.

[4] David, S. and Edlich, S. 2009. A location-based notificationAnd visualization-system indicating social activities. Proceedings of SPIE - The International Society for Optical Engineering, 7256.

[5] DeVaul, R.W., Sung, M., Gips, J., and Pentland, S. 2003. MIThril 2003: Applications and Architecture. In Proceedings of ISWC 2003 (White Plains, USA, 2003).

[6] Elias, R. and Elnahas, A. 2009 Fast localization in indoor environments. Proceedings of the 2009 IEEE Symposium on computational intelligence, CISDA, 1-6.

[7] Gravina, R., Guerrieri, A., Fortino, G., Bellifemine, F.., Giannantonio, R. and Sgroi, M. 2008. Development of body sensor network applications using SPINE. In Proceedings of the IEEE International Conference on Systems, Man and Cybernetics (SMC 2008 Singapore Oct 2008) 2810-2815.

[8] Hesch, J. A., Mirzaei, F.M., Mariottini, G.L., and Roumeliotis, S.I. 2009. A 3D pose estimator for the visually impaired. IEEE/RSJ International Conference on Intelligent Robots and Systems, IROS 2009, 2716-2723.

[9] Jovanov, E., Milenkovic, A., Otto, C., and de Groen, P.C. 2005. A wireless body area network of intelligent motion sensors for computer assisted physical rehabilitation. Journal of NeuroEngineering and Rehabilitation 2, 6.

[10] Kang, H., Efros, A.A., Hebert, M., and Kanade, T. 2009. Image matching in large scale indoor environment. IEEE Conference on Computer Vision and Pattern Recognition, CVPR 2009, 33-40.

[11] Kuryloski, P., Giani, A., Giannantonio, R., Gilani, K., Gravina, R., Seppa, V., Seto, E., Shia, V., Wang, C., Yan, P., Yang, A.Y., Hyttinen, J., Sastry, S., Wicker, S., and Bajcsy, R. 2009. DexterNet: An Open Platform for Heterogeneous Body Sensor Networks and its Applications. Body Sensor Networks 2009 (BSN 2009 Berkeley, CA June 3-5, 2009) 92-97.

[12] Lorincz, K., Malan, D.J., Fulford-Jones, T.R.F., Nawoj, A., Clavel, A., Shnayder, V., Mainland, G., Welsh, M., and Moulton, S. 2004. Sensor Networks for Emergency Response: Challenges and Opportunities. IEEE Pervasive Computing, 3, 4, 16-23.

[13] Ryder, J., Longstaff, B., Reddy, S., and Estrin, D. 2009. Ambulation: A tool for monitoring mobility patterns over time using mobile phones, Proceedings - 12th IEEE International Conference on Computational Science and Engineering, CSE 2009, 4, 927-931.

[14] Seppä, V., Väisänen, J. , Lahtinen, O., and Hyttinen. J. 2009. Assessment of breathing parameters during running with a wearable bioimpedance device. In Proceedings of 4th European Conference of the International Federation for Medical and Biological Engineering. (ECIFMBE 2008 2327 November 2008 Antwerp, Belgium) 1088-1091.

[15] Seto, E.Y.W., Giani, A., Shia, V., Wang, C., Yan, P., Yang, A.Y., Jerrett, M., and Bajcsy, R. 2009. A Wireless Body Sensor Network for the Prevention and Management of Asthma, IEEE International Symposium on Industrial Embedded Systems, 2009 July 8-10. 120 - 123.

[16] Shayeganfar, F., Anjomshoaa, A., and Tjoa, A.M. 2008. A smart indoor navigation solution based on building information model and Google Android. Lecture Notes in Computer Science, 5105 LNCS, 1050-1056.

[17] Xianhua, S., Zhenjun, D., and Rong, C. 2009 Research on mobile location service design based on Android. Proceedings - 5th International Conference on Wireless Communications, Networking and Mobile Computing, WiCOM 2009.

[18] Yan, P., Lin, I., Roy, M., Seto, E., Wang, C., and Bajcsy, R. 2010. WAVE and CalFit - Towards Social Interaction in Mobile Body Sensor Networks. In Proceedings of the 2010 International Workshop on Ubiquitous Body Sensor Networks UBSN 2010 (Singapore, March 1-3, 2010). 
[19] Yang, A., Jafari, R., Sastry, S., and Bajcsy, R. 2009. Distributed Recognition of Human Actions Using Wearable
Motion Sensor Networks. Journal of Ambient Intelligence and Sensor Environments (JAISE) 1, 2, 103-115. 